\title{
Power Coordinated Control of Micro Sources of MMC Series Microgrid Based on Model Prediction
}

\author{
Yanni Ma ${ }^{1}$, Xinggui Wang ${ }^{1, *}$ and ShengXue ${ }^{1}$ \\ ${ }^{1}$ College of Electrical and Information Engineering, Lanzhou University of Technology, Lanzhou, Gansu, 730050, China
}

\begin{abstract}
To improve the utilization rate of micro sources of MMC series structured microgrid in a gridconnected operation mode, a prediction-based coordination control strategy of micro-source power of bridge arm is proposed in this paper. The connection between the output power and the delta carrier is determined by analyzing each generation unit's output power. Each micro source's output power is controlled independently by using the carrier-wave variable amplitude phase-shift modulation method. A finite control set-model predictive control algorithm is used to predict the next cycle's power using the carrier amplitude of the current cycle as the controlled input. The optimal switching state for the next cycle is determined according to the coordination principle of "more work for more energy." Through simulation, the coordination strategy can effectively control the maximum power output of the bridge arm and improve the micro source's utilization rate.
\end{abstract}

\section{Introduction}

In the research of various control technologies of microgrids, coordinated control technology occupies an important position. In the grid-connected mode, the purpose of the microgrid's power coordination is to maintain the stable transmission of energy between the microgrid and the grid, and to improve the system's operating efficiency [1]. For the AC microgrid with a parallel structure of multi-micro-source inverters, most of them use multi-agent-based distributed control, hierarchical control, and peer-to-peer control to coordinate and distribute the micro-sources [2-4]. However, the parallel structure AC microgrid has problems such as large circulating current and severe harmonic pollution to the distribution network. Literature [5] proposed an MMC series structure microgrid (Modular Multilevel Converter Microgrids, MMC-MG), the aim is to solve some of the problems mentioned above from the structural aspect. Compared with the traditional parallel microgrid structure, MMCMG has the advantage of flexibly controlling the switching of sub-modules, making the system output voltage level higher and the harmonic content smaller. Hence, it is more conducive to grid-connected operation. Since each micro-source in the MMC-MG's bridge arm is contained in a power generation unit, each power generation unit is a series structure, the traditional microgrid's micro-source power coordination method is not applicable. Literature [6] introduces a model predictive control method for the MMC structure, aiming to reduce the control error of its capacitor voltage and improve the control performance. To improve the utilization of MMC-MG's micro-sources and improve its coordinated control performance, it is indispensable to study the power coordinated control method of the bridge arm's micro-sources in the grid-connected mode.

To reduce the error generated in the coordinated control of micro-source power, this paper adopts the finite control set-model predictive control (FCS-MPC) algorithm to predict the next cycle's output power in advance. The minimum evaluation function is selected to improve the accuracy of prediction. The minimum evaluation function was selected to improve the accuracy of the prediction. The predicted power values were sorted to ensure the micro-source input with large output combined with the coordination principle of "more work for the good." Using the carrier variable amplitude and phase shift modulation method, the output power's expected value is converted into the corresponding triangular carrier amplitude to realize the independent adjustment of each micro source's output power, thereby controlling the micro source with the considerable output power in each cycle. Finally, simulation proves the effectiveness of the control strategy.

\section{MMC-MG topology and output power analysis}

The MMC-MG system is a three-phase system, and each phase has the same structure, divided into two upper and lower bridge arms. Each bridge arm comprises $N$ power generation units (Generate Modulation, GM) and one inductor $L$. The inverter link is formed by the MMC topology structure, connected to the external power grid and $\mathrm{AC}$ load through the filter, and the MMC-MG gridconnected topology structure as shown in Figure 1. 
GM is composed of micro-sources such as photovoltaic cells and wind turbines. It is connected in parallel to the DC side of a half-bridge converter (HC) through an AC-DC conversion circuit. The purpose of connecting to the energy storage device (ES) is to smooth out voltage fluctuations and improve the system. The reliability and diversity of power supply. The structure of GM is shown in Figure 2.

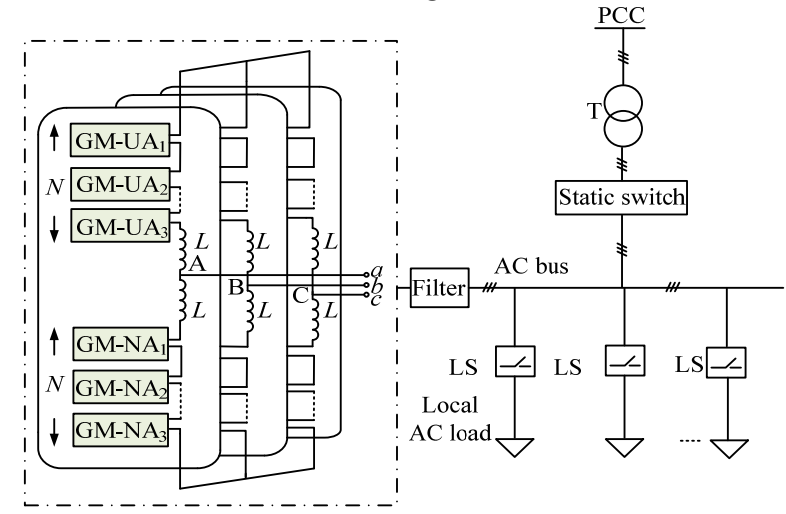

Fig. 1. Grid-connected MMC-MG topology

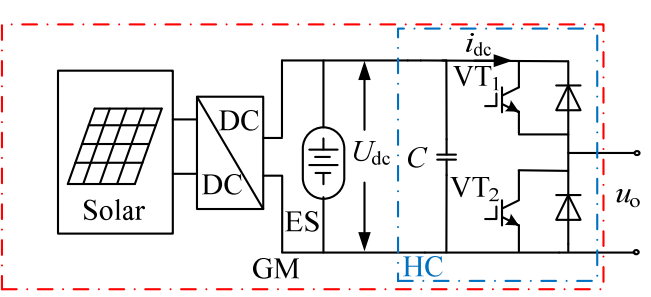

Fig. 2. (a) Photovoltaic GM structure

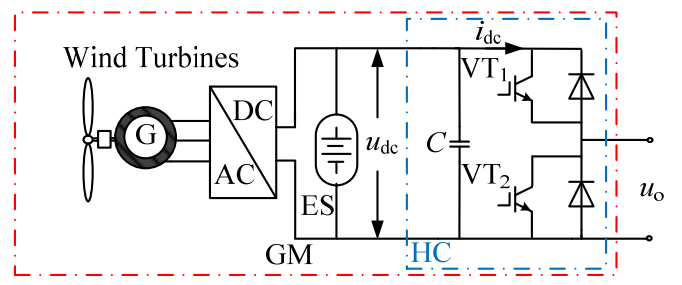

Fig. 2. (b) Fan GM structure

The MMC-MG equivalent circuit diagram in gridconnected mode is shown in Figure 3. MMC-MG has no common DC bus voltage, so $U_{\mathrm{zd}}$ is the virtual DC bus voltage and $R$ is the equivalent resistance of the bridge arm. The output voltage of each GM can be identical to a controlled voltage source.

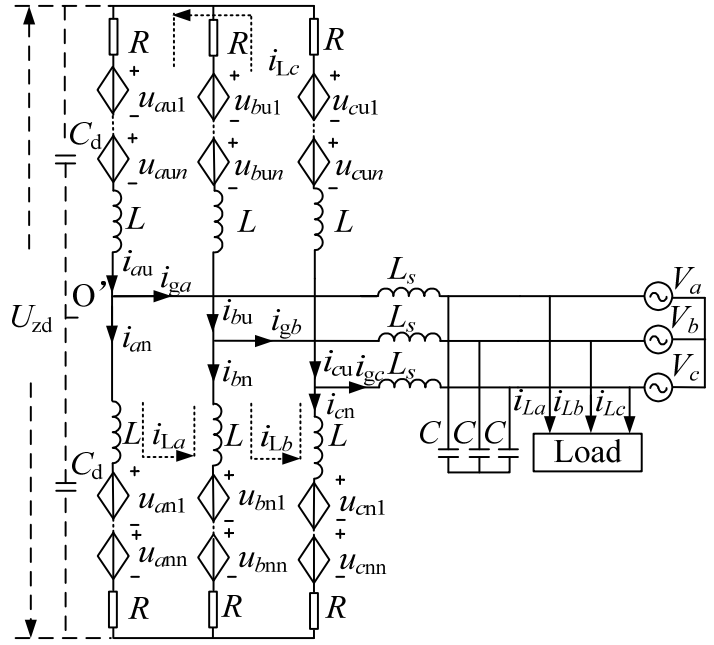

Fig. 3. Grid-connected MMC-MG equivalent circuit

Due to the three-phase symmetry, taking Phase A as an example, the affective component of the GM output power is the DC component, which can be obtained from the literature [5]:

$$
P_{a u i_{-} \mathrm{dc}}=\frac{u_{\text {daui }}}{2}\left[\frac{m_{a i}}{2}\left(I_{g a m} \frac{\cos \varphi}{2}+I_{a l 1} \cos \varphi_{a l 1}\right)-I_{a l_{-} \mathrm{dc}}\right]
$$

In the equation (1), $u_{\text {daui }}$ and $m_{a i}$ are the ith GM's DC side voltage and modulation ratio; $I_{\text {gam }}$ is the A-phase current amplitude of the power grid; $I_{l a}$ dc and $I_{a l 1}$ are the DC component and the fundamental frequency component in the circulating current component, respectively; $\varphi$ is the power factor angle; $\varphi_{a l 1}$ is the initial angle of the fundamental frequency component of the circulating current.

When the system is running, if the circulating current is suppressed and $i_{a l 1}$ is negligible compared to the current grid $I_{g a}$, the DC component of the instantaneous output power of GM as:

$$
P_{a u i_{-} \mathrm{dc}}=m_{a i} \frac{u_{\text {daui }}}{2} \frac{I_{\text {gam }}}{2} \frac{\cos \varphi}{2}
$$

The modulation ratio of each GM in the bridge arm is defined by

$$
m_{a 1}+m_{a 2}+\cdots+m_{a N}=N m
$$

Equation (3) is a constraint condition to ensure that the system's output voltage meets the demand. In the equation, $m_{a i}$ represents the modulation ratio of the ith $\mathrm{GM} ; m$ is the average modulation ratio of system.

\section{Principle of Micro-source Power Coordination}

In grid-connected mode, the maximum power point (MPP) of each micro source should be ensured. On this basis, the unit with the maximum output power is selected among all GMs and put into operation so that the three-phase system can transmit the maximum power 
to the grid. Taking any phase as an example, the output power of the bridge arm should be defined by

$$
P=\max \sum_{i=1}^{N} s_{i} P_{\mathrm{GM} i}
$$

In the equation(4), $P_{\mathrm{GM} i}$ is the output power value of the ith GM at the MPP point; $s_{i}$ is the GM switching judgment function, and the value of $s_{i}$ is 0 or 1, taking 0 to indicate that the GM is in the removal state, and taking 1 to suggest that the GM is in the on-state.

From equation (2), we can see that $P_{a u i \_d c} \propto m_{a i}$. The modulation ratio is the ratio of the amplitude of the modulating wave to the amplitude of the triangular carrier, so by adjusting the corresponding amplitude of each triangular carrier, the independent control of the output power of each micro-source can be realized.

Take the A-phase upper bridge arm containing 4 GMs as an example, and each GM corresponds to a triangular carrier. Suppose $u_{c 2}$ is the reference amplitude, $\left|\Delta u_{i}\right|$ is the amplitude change of the triangular wave, and the principle diagram of carrier variable amplitude and phase shift modulation is shown in Figure 4.

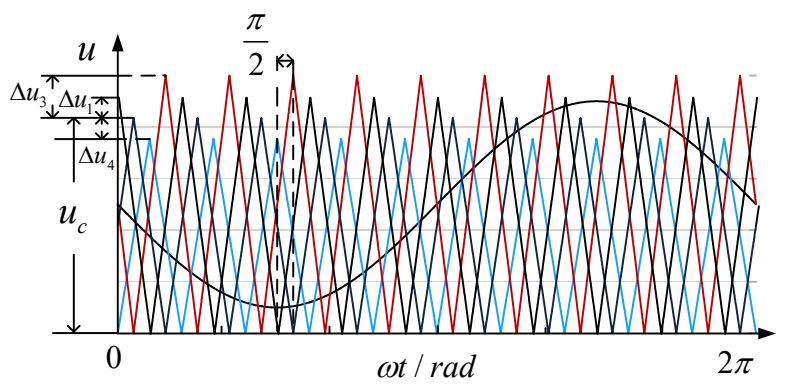

Fig. 4. Schematic diagram of carrier variable amplitude and phase shift

The ratio of each GM's output power is equal to the ratio of the corresponding modulation ratio. Take the amplitude of the modulation wave and the basic amplitude of the triangular carrier as 1 , then there is given by

$$
\begin{aligned}
& \left|P_{\mathrm{GM} 1}\right|:\left|P_{\mathrm{GM} 2}\right|:\left|P_{\mathrm{GM} 3}\right|:\left|P_{\mathrm{GM} 4}\right|=\frac{1}{1+\left|\Delta u_{1}\right|} \\
& : \frac{1}{1+\left|\Delta u_{2}\right|}: \frac{1}{1+\left|\Delta u_{3}\right|}: \frac{1}{1+\left|\Delta u_{4}\right|}
\end{aligned}
$$

\section{Micro-source power coordinated control strategy}

To improve the coordination performance, the FCSMPC is combined with the carrier variable amplitude and phase shift modulation strategy. FCS-MPC is an algorithm in model predictive control, characterized by being able to follow the real-time changes of the system in time and obtain the control scheme online [7].

The triangular carrier amplitude set corresponding to the bridge arm GM is $u(k) \in\left\{u_{1}(k), u_{2}(k), \ldots, u_{n}(k)\right\}$, which constitutes a Finite Control-Set (FCS). The output power of each GM can be adjusted independently by adjusting the amplitude of each triangular carrier.

The prerequisite for the realization of FCS-MPC is to establish a discrete mathematical model [8]. The discrete mathematical model of the GM output power in the MMC-MG phase can be expressed as:

$$
P_{\mathrm{GM}}(k+1)=\Gamma P_{\mathrm{GM}}(k)+\mathrm{K} u_{c}(k)
$$

In the equation(6), $P_{\mathrm{GM}}(k+1)$ is the predicted value of the GM output power in the $k+1$ cycle; $P_{\mathrm{GM}}(k)$ is the sampled value of the GM output power in the k-th cycle; $u_{c}(k)$ is the triangular carrier amplitude of the $\mathrm{k}$-th cycle; $\Gamma \approx I+A T_{s} ; \mathrm{K} \approx B T_{s}, T_{\mathrm{s}}$ is the sampling cycle of the system, $I$ is the identity matrix, and $A$ and $B$ are the parameter matrices.

To further improve the accuracy of prediction, the evaluation function corresponding to coordinated control

can be expressed as:

$$
g=\left|P_{G M}^{*}(k+1)-P_{G M}(k+1)\right|
$$

In the equation(7), $P_{G M}^{*}(k+1)$ is the reference value of the GM output power in the $k+1$ cycle. Choose the corresponding GM with the minor evaluation function to reduce the prediction error.

Since the output of intermittent micro-sources will be affected by environmental factors and change, each GM's output power is different, with different power reference values, and reference extrapolation technology is adopted to solve this problem. The corresponding Lagrangian extrapolation of the two-step prediction $P_{G M}^{*}(k+2)$, which is performed by equation (8)[9].

$$
\begin{aligned}
& P_{G M}^{*}(k+2)=\sum_{l=0}^{n}(-1)^{n-l} \frac{(n+1) !}{l !(n+1-l) !} P_{G M}^{*}(k \\
& +1+l-n)
\end{aligned}
$$

In the equation(8), $n$ represents the order of extrapolation. In this paper, $n$ takes 2.

The GM output power reference value corresponding to the $k+1$ cycle is expressed by equation (9).

$$
\begin{aligned}
& P_{G M}^{*}(k+1)=4 P_{G M}^{*}(k)-6 P_{G M}^{*}(k-1)+4 P_{G M}^{*}(k-2) \\
& -P_{G M}^{*}(k-3)
\end{aligned}
$$

The GM output power in the $k+1$ cycle is predicted according to the GM output power's sampled value in the $k$ cycle and its control variables. On the one hand, the switching state of each GM in the $k+1$ cycle is determined by a sorting algorithm; on the other hand, combined with carrier variable amplitude and phase shift modulation, the strategy is to obtain the $k+1$ periodic triangular carrier amplitude through equations (2) and (6) and independently control the output power of each GM. Besides, the predicted value of the $k+2$ th cycle can be obtained by equation (8). The principle of power coordination based on FCS-MPC is shown in Figure 5. 


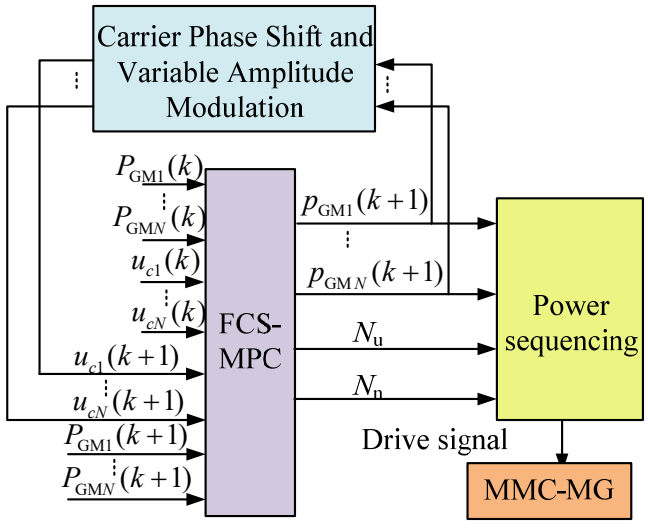

Figure 5. Power coordination principle based on FCS-MPC

Determine the power interval $\left[P_{\mathrm{GM}, \min }, P_{\mathrm{GM}, \max }\right]$ through the modulation ratio's adjustment range, which is the controllable interval of the GM output power.

$$
\left\{\begin{array}{l}
P_{\mathrm{GM}, \text { max }}, P_{\mathrm{GM}, \text { max }}<P_{\mathrm{GM}} \\
P_{\mathrm{GM}}, P_{\mathrm{GM}, \text { min }}<P_{\mathrm{GM}}<P_{\mathrm{GM}, \max } \\
P_{\mathrm{GM}, \text { min }}, P_{\mathrm{GM}, \text { min }}>P_{\mathrm{GM}}
\end{array}\right.
$$

In the above equation, when the predicted power exceeds the power interval, if it exceeds the upper limit of power, the output power is maintained; if the output power is less than the lower limit of power, the output power is maintained. When the predicted power is within the power range, no correction is made. To ensure that only N GMs are put into any phase simultaneously, the GM power coordination flow diagram is shown in Figure 6.

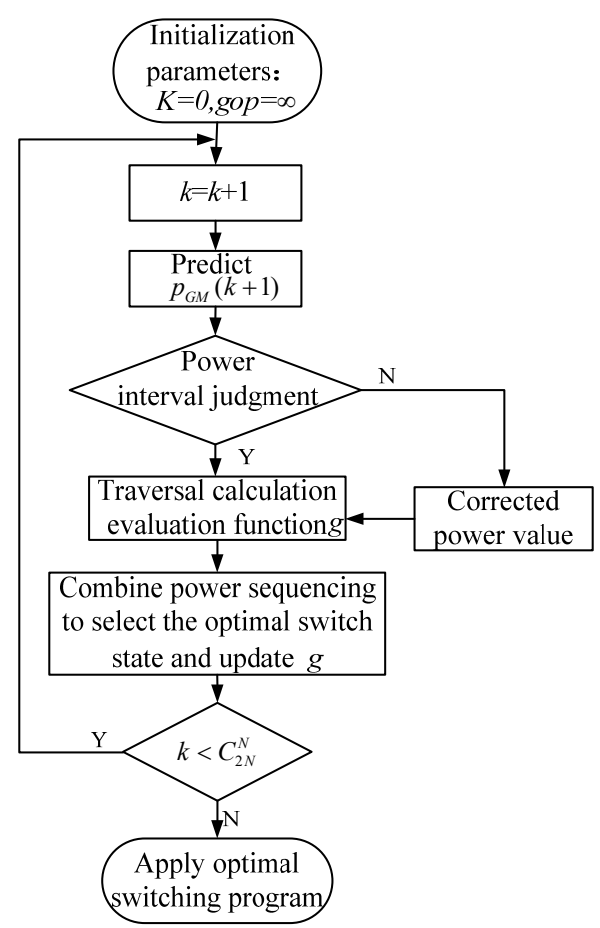

Fig. 6. Block diagram of power coordination process

\section{Simulation and analysis}

To verify the coordinated control strategy, a simulation model was built. The number of micro-sources in the bridge arm is set to 4 , and the system simulation parameters are shown in Table 1 .

Table 1. System simulation parameter

\begin{tabular}{|c|c|c|c|c|c|}
\hline parameter & $f_{c} / \mathrm{kHz}$ & $C / \mu \mathrm{F}$ & $L_{s} / \mathrm{mH}$ & $R_{S} / \Omega$ & $T_{s} / \mathrm{ms}$ \\
\hline value & 1 & 4400 & 5 & 0.18 & 20 \\
\hline
\end{tabular}

To study the adjustment range of the carrier variable amplitude and phase shift modulation strategy to the $P_{\mathrm{GM}}$, take $m_{i} \in(0.54,0.98)$ and set the modulation ratio of each GM to 0.54 and 0.98 , respectively. The simulation results are shown in Figure 7.

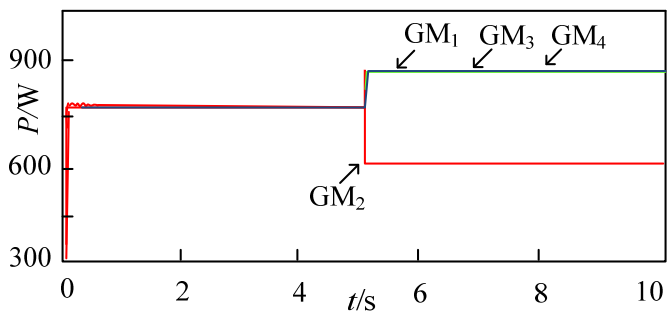

Fig. 7. Power adjustment range diagram

It can be seen from Figure 7 that within $0 \sim 5 \mathrm{~s}$, when $m_{1}=m_{2}=m_{3}=m_{4}=0.98$, the output power of each GM reaches $790 \mathrm{~W}$ at this time; in $5 \sim 10 \mathrm{~s}, m_{2}$ is set to 0.56 , at this time the output power of $\mathrm{GM}_{2}$ down to $610 \mathrm{~W}$, $P_{\mathrm{GM} 1}=P_{\mathrm{GM} 3}=P_{\mathrm{GM} 4}=850 \mathrm{~W}$, thus the power adjustment range can be $240 \mathrm{~W}$. When the modulation ratio is changed, the corresponding carrier variable amplitude and phase shift modulation diagram is shown in Figure 8.

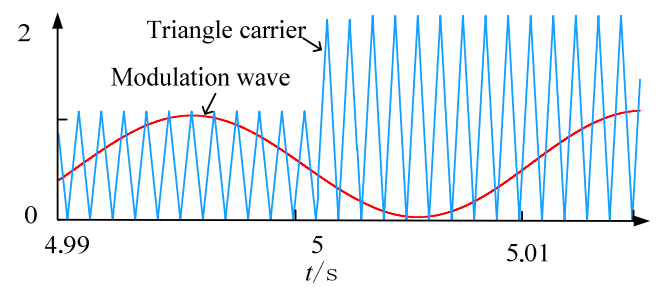

Fig. 8. Carrier Variable Amplitude and Phase Shift Modulation

Taking the upper bridge arm of phase $\mathrm{A}$ as an example, $\mathrm{GM}_{1}$ and $\mathrm{GM}_{2}$ contain photovoltaic micro sources; $\mathrm{GM}_{3}$ and $\mathrm{GM}_{4}$ have fan micro sources. At $1.5 \mathrm{~s}$, the light intensity is reduced, and the wind speed is increased, and the corresponding output is shown in Figure 9. 


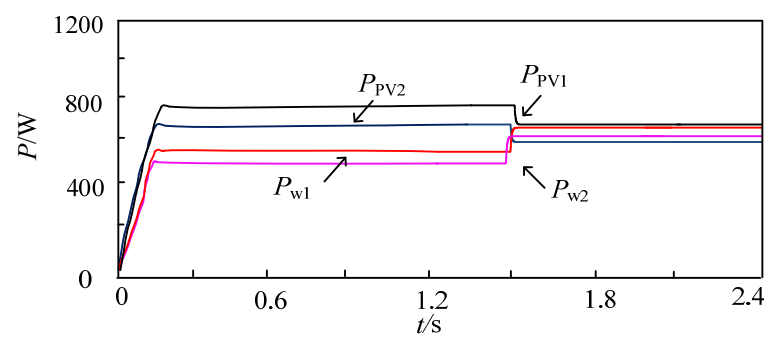

Fig. 9. GM collects real-time power when the light intensity and wind speed change

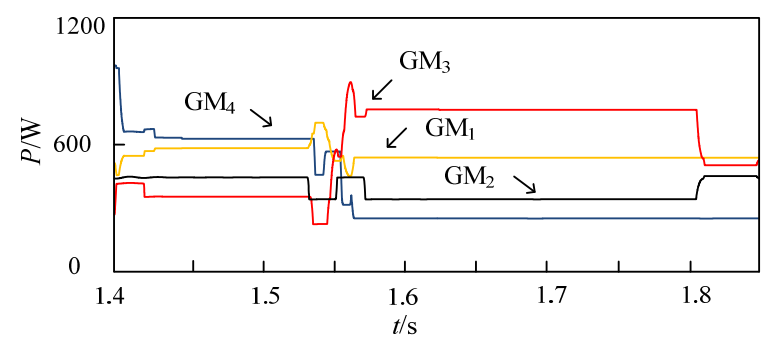

Fig. 10. Real-time collection of coordinated control waveforms of GM output

The output power of each GM when FCS-MPC is not used for power coordinated control is shown in Figure 10. It can be seen that when FCS-MPC is not used for power coordination, the GM output power fluctuates many times. Especially after the $1.5 \mathrm{~s}$ micro-source light intensity and wind speed change, the GM output magnitude sequence fails to change in time. Still, it goes through, and it only starts to change about $0.5 \mathrm{~s}$, indicating that the control effect is not ideal. After $1.8 \mathrm{~s}$, it was not put into operation according to the power sequencing results, resulting in insufficient micro-source utilization.

After using FCS-MPC for power coordination, the output power of each GM is shown in Figure 11. It can be seen from Figure 11 that before $1.5 \mathrm{~s}$, the output power of each GM has no fluctuations. Among them, the output power of $\mathrm{GM}_{3}$ and $\mathrm{GM}_{4}$ is outside the poweradjustable range, so it is maintained at the lower limit of the revised power range. After the $1.5 \mathrm{~s}$, micro-source light intensity and wind speed change, the GM that with the maximum output power according to the coordination principle is put into operation, and $\mathrm{GM}_{1}$ is within the adjustable range; after $\mathrm{GM}_{2}$ reduces the light intensity, the output power is lower than the lower limit of power at this time. After a short drop, it is maintained at the lower limit of the power interval.

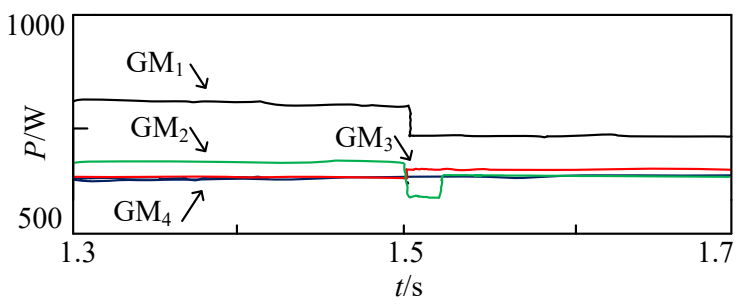

Fig. 11. Power Coordinated Control Waveform Based on FCSMPC

\section{Conclusion}

This paper studies a power coordinated control method of micro-sources for MMC-MG in grid-connected operation mode. FCS-MPC is used to improve the performance of coordinated control. The carrier variable amplitude and phase-shift modulation strategy are combined with coordinating power between micro sources. The simulation results show that the coordination method can independently control the micro-source operation with the most considerable output power without affecting the output of other micro-sources and improving the utilization of the micro-sources. After changing the micro-source light intensity, wind speed, and other conditions, each GM can quickly and stably switch on the principle of microsource coordination, reducing the control error. The coordinated control method lays the foundation for the power coordination of the MMC-MG system, and the power coordinated control method of the system can be further studied.

\section{Acknowledgments}

This paper is one of the phased achievements of the National Natural Science Foundation of China project Research on the Key Technology of Microgrid System Based on MMC Half-Bridge Micro-source Inverter Series Structure.(No.51967011)

\section{References}

1. S.S. Yang, P.P. Luo, (2019) Research on Power Coordinated Control Strategy of Photovoltaic Gridconnected System with Hybrid Energy Storage. Modern power, 36(01):37-44.

2. M. Ding, K. Luo, R.Bi, (2013) Multi-agent systembased energy coordinated control strategy for microgrid in island mode. Automation of Electric Power Systems, 37(05):1-8+43.

3. X. Xiong, M. Wu, Y. Ji, et al. (2018) Research on Hybrid Coordinated Control and Power Management Strategy of Microgrid Group. Chinese Society for Electrical Engineering, 38(05):14191427.

4. X.P. Zhou, Y.D. Chen, L.M. Zhou, et al. (2017) A microgrid group architecture and its autonomous coordinated control strategy. Journal of Electrotechnical Technology, 32(10):123-134.

5. X.G. Wang, S. Xue, X.Y. Li, (2019) Analysis of output characteristics of modular multilevel converters in series with half-bridge microgrid. Journal of Electrotechnical Technology, 34(10):2130-2140.

6. F. Peng, Y.F. Li, Z.W. Bu, et al. (2017) MMC capacitor voltage control strategy based on FCSMPC control algorithm. Power Electronics Technology, 51(06):64-66. 
7. X.Q. Wang, J.W. Zhao, Q.J. Wang, et al. (2019) Fast FCS-MPC-Based SVPWM Method to Reduce Switching States of Multilevel Cascaded H-Bridge STATCOMs. JOURNAL OF POWER ELECTRONICS, 19(1).

8. D.H. Zhou, et al. (2019) Finite-control-set model predictive control of modular multilevel converters with cascaded open-circuit fault ride-through. IEEE Journal of Emerging and Selected Topics in Power Electronics, 8(3): 2943-2953.

9. Y. Xu, X.Y. Pan, X.Niu ,et al. (2015) Microgrid energy management optimization using model predictive control: a case study in China. IFACPaperOnLine, 48(30): 306-311 